\title{
Follow-up examinations performed by radiation oncologists after radiotherapy in Japan
}

\author{
Osamu Tanaka ${ }^{*}$, Eiichi Yama ${ }^{2}$, Hironori Ichihashi ${ }^{2}$, Yuzo Yamada ${ }^{2}$, Junichi Ando ${ }^{2}$ and Takayoshi Iida ${ }^{1}$ \\ ${ }^{1}$ Department of Radiation Oncology, Gifu Municipal Hospital, 7-1 Kashima-cho, Gifu City, Gifu, Japan \\ ${ }^{2}$ Division of Radiation Service, Gifu Municipal Hospital, 7-1 Kashima-cho, Gifu City, Gifu, Japan
}

\begin{abstract}
Aim: To know how many patients desire re-examination in radiotherapy department.

Background: As there are few radiotherapists in Japan, it is impossible to perform post-radiotherapy examinations in some cases. we conducted a questionnaire-based survey to discover whether patients desired to undergo follow-up examinations at the radiation oncology department in addition to the examinations performed by the attending physician after radiotherapy and to discover the types of patients who desired such examinations.

Materials and methods: We conducted a questionnaire-based survey of 109 patients to investigate the number of patients who desired to undergo follow-up examinations at the radiation oncology department similar to those received at the department of their attending physician.

Results: Forty-three percentage of the patients desired follow-up examinations. A statistically significant number of men reported that they desired follow-up examinations at both departments. Patients with brain tumors did not desire follow-up examinations at both departments. There was a great individual variation in the desire for follow-up examinations at the radiation oncology department.
\end{abstract}

Conclusion: As a whole, $43 \%$ of patients hope for the second visit. It is necessary to repair these present conditions in Japan.

\section{Introduction}

Radiotherapy is one of the three most commonly performed major cancer treatments, and the number of patients receiving radiotherapy is expected to increase in the future. However, as the number of radiotherapists is smaller in proportion to the number of patients, handling this increased demand will pose a problem [1-3].

In most cases, radiation oncology departments in hospitals work with the referral department for the treatment of patients, rarely acting independently in the role of attending physician. Thus, the implementation of post-radiotherapy examinations greatly varies, particularly in facilities where there are only part-time physicians or only one full-time physician, and the patient-to-physician ratio is overwhelmingly out of balance, thereby contributing to the uneven implementation of post-radiotherapy examinations.

In many cases, patients are asked by their attending physician to continue outpatient follow-up examinations for several years after treatment, and patients often find this difficult and burdensome. There are also patients who find it annoying to have to relay the conversations they had with their attending physician (the referring physician) to the radiation oncology department. Thus, in the present study, we conducted a questionnaire-based survey to discover whether patients desired to undergo follow-up examinations at the radiation oncology department in addition to the examinations performed by the attending physician after radiotherapy and to discover the types of patients who desired such examinations.

\section{Methods and subjects}

The subjects were 109 consecutive patients who underwent radiotherapy between October 2015 and March 2016. The study was approved by the institutional review board (approval number: 272) of this hospital and UMIN-ICTR (UMIN000019404). Written consent was obtained from all subjects. None of the patients refused to respond to the questionnaire.

Forty hundred and fifty patients are treated at my hospital a year. A patient receiving treatment using IMRT is approximately $40 \%$. My hospital is secondly a big hospital in a prefecture. (our hospital; one radiation oncologist, four radiotherapists and one nurse.) Currently, patients undergo examinations at the radiation oncology department only during radiotherapy at our hospital. All patients undergo postradiotherapy follow-up examinations at the referral departments.

Medical examinations and radiotherapy regimens at our institution were developed by a radiation oncologist with 20 years of experience. There was no medical physicist at our hospital, but there were four radiological technicians; the radiotherapy devices used included Novaris Tx and Clinac. We see 450 patients per year at our hospital and administer intensive modulated radiation therapy (IMRT) when required for the head and neck, abdomen, and pelvis. We also administer stereotactic radio surgery (SRS) for the brain and stereotactic body radiation therapy (SBRT) for the lungs and liver.

Correspondence to: Osamu Tanaka, MD, Department of Radiation Oncology, Gifu Municipal Hospital, 7-1 Kashima-cho, Gifu City, Gifu, Japan, Tel: 0582511101; Fax: 0582521335; E-mail: c.bluered@gmail.com

Key words: radiotherapy, follow-up examinations, radiation oncologist, Japan

Received: May 04, 2017; Accepted: May 17, 2017; Published: May 22, 2017 
Patients were asked to complete the questionnaire shown in Table 1. Because the handing out of questionnaires by staff members wearing white lab coats could lead to a bias, we asked the reception staff of the outpatient department to hand them out to patients a week before their treatment was scheduled to end. We performed a chi-square test of pearson. P value did 0.05 or less if significantly different.

\section{Results}

There were $43 \%$ of patients desire re-examination in our department.

Table 2 shows that significantly greater number of men desired follow-up examinations $(\mathrm{p}=0.04)$, whereas a significantly greater number of brain tumor patients did not desire follow-up examinations $(\mathrm{p}=0.04)$.

No other types of patients had factors with statistically significant differences.

\section{Discussion}

The benefit of being examined at the radiation oncology department

Table 1. Questionnaire regarding post-radiotherapy follow-up examinations.

Currently, radiation oncologists perform initial examinations and mid-treatment examinations of patients undergoing radiotherapy, and referring physicians in the diagnostic and treatment departments (attending physicians) observe their post-treatment progress.

As discussed at the initial examination, the effects of radiation can appear several months after treatment completion. Therefore, please circle any one of the following two options regarding your desire to undergo a follow-up examination after treatment completion:

1) I believe a post-radiotherapy examination by my attending physician is sufficient.

2) I desire a follow-up examination by a radiation oncologist.

Please note that your refusal to participate in this questionnaire will not hamper your treatment.

Table 2. Questionnaire regarding the desire for follow-up examinations after the completion of radiotherapy.

\begin{tabular}{|l|c|c|}
\hline Total no. of patients & 109 & $P$ value \\
\hline Desire & 43 & \\
\hline Do not desire & 66 & $0.04^{*}$ \\
\hline Gender & Desire/total no. & \\
\hline Male & $31 / 69$ & \\
\hline Female & $12 / 40$ & 0.11 \\
\hline Mean age (years) & 69.9 & 0.98 \\
\hline Irradiation objective & & \\
\hline Cured & 28 & \\
\hline Palliated & 51 & 0.22 \\
\hline Mean dose (Gy) & 53 & \\
\hline Anatomical location of irradiation & Desire/total no. & $0.04 *$ \\
\hline Brain & $0 / 6$ & 0.28 \\
\hline Head/neck & $3 / 12$ & 0.7 \\
\hline Breast & $7 / 16$ & 0.74 \\
\hline Lung & $8 / 16$ & 0.82 \\
\hline Abdomen & $5 / 10$ & 0.87 \\
\hline Prostate & $7 / 16$ & 0.32 \\
\hline Pelvis & $4 / 5$ & 0.97 \\
\hline Bone (incl. metastasis) & $4 / 10$ & 0.45 \\
\hline Other & $5 / 18$ & \\
\hline A & & \\
\hline
\end{tabular}

A significantly greater number of men desired follow-up examinations.

A significantly greater number of brain cancer patients did not desire follow-up examinations.

Brain: total brain irradiation and stereotactic radio surgery (SRS)

Abdomen: liver cancer, pancreatic cancer, and gallbladder cancer

Pelvis: pelvic tumors excluding those of the prostate (colorectal cancer, obstetric tumors, and bladder cancer).

Other: any type that had four or fewer patients was classified as "Other"; hematological malignancy, benign neoplasm, skin cancer, soft tissue neoplasm, and lymph node metastases is that the radiologist who planned the radiotherapy is most familiar with the extent and dose of radiation used and is best able to anticipate the extent and location of adverse effects. The radiation oncology department is able to anticipate the chronological effects of these factors over the course of treatment. Post-treatment examinations serve as a learning tool because they allow radiation oncologists to follow the course of the patient's progress, allowing them to determine if the treatment was accurate. Thus, from the radiation oncologist's perspective, follow-up examinations are the most advantageous tool.

However, insufficient number of radiation oncologists in Japan is a serious problem, and from a manpower perspective, it seems unlikely that this problem will resolve in the near future. According to Nishie's survey [4], in Japan, the total number of radiation oncologists is 732 . Among them, 664 responded in regard to their employment status: 497 were full-time (74.8\%), 167 part-time, and 68 unknown. Although the number of radiation oncologists is gradually increasing, this increase is not at approximately the same pace at which the number of patients is increasing.

One of the late effects of radiotherapy for breast cancer is coughing caused by radiation pneumonia, and breast surgeons are familiar with this phenomenon. Similarly, urologists are often aware of the fact that rectal hemorrhage is a late effect of radiotherapy for prostate cancer. Thus, when patients receive the same explanation from multiple physicians, it just adds to the number of outpatient visits and medical cost burdens; this is probably why the results of this study indicate that $57 \%$ of patients do not desire follow-up examinations at the radiation oncology department. Although the radiation oncology department at our hospital provides explanations for the late effects of radiotherapy during both the initial examination and course of treatment, many patients think that the explanation received from the attending physician is sufficient, because in many cases, they have already previously heard an overview of the radiotherapy.

The preferences of patients at our hospital are not necessarily applicable to or predictive of practices at other centers and regions in Japan; however, according to our survey, a significantly greater number of women said that they desired to undergo follow-up examinations. We considered the possible influence of diseases peculiar to women, such as breast cancer, but none of these showed a statistically significant correlation with the female sex.

In this study, we did not distinguish between outpatients and inpatients. Although in many cases, hospitalized patients receive more intense treatment and have more advanced forms of cancer, the desire for follow-up examinations showed a great individual variation, making it impossible for us to consider outpatients and inpatients as distinct groups. If we could prove that the treatment result (tumor control rate, complication rate, quality of life, medical economy, patient's mental status, etc.) becomes worse if radiation oncologists do not follow-up, our data becomes very valuable and can influence the present situation of Japan.

Although our data is small and ambiguous, the results of this study indicate that $43 \%$ of patients desire to undergo follow-up examinations. Therefore, it is necessary for the small number of Japanese radiation oncologists to create an environment that facilitates follow-up examinations. At present, in this study, we are afraid that $43 \%$ of patients desired follow-up examinations at our hospital; however, $80 \%$ may desire the examination in hospital A (or Dr. A) and $10 \%$ in hospital B (or Dr. B). The results may greatly fluctuate depend on the physician's examination technique. 
For example, it may be necessary for radiation oncologists to consult with patients when their treatment is completed in order to set up a follow-up examination schedule. As some patients may find it difficult to refuse the follow-up examination recommended by the physician, we believe it is important that staff members who are not wearing lab coats discuss follow-up examinations with patients in a flexible manner and in accordance with the patients' responses to the questionnaire administered toward the end of their treatment.

\section{Conclusion}

There was a great individual variation in the desire for follow-up examinations at the radiation oncology department (43\%). Accordingly, we believe that as part of the responsibilities of radiation oncologists, it is important to employ follow-up examinations to observe the progress of such patients.

\section{Acknowledgement}

We thank Mrs. Kimiko Sawai to help medical data sampling.

Disclosure of potential conflicts of interest; None Research involving Human Participants and/or Animals; Yes belowWe received written Informed consent from all patients.

\section{References}

1. Japanese PCS Working Group (2006) JASTRO Fiscal 2003/2004 Research Topics Report, Revision of guideline for structure of radiation oncology by the patterns of care study. J Jpn Soc Ther Radiol Oncol 18: 107-112.

2. Matsumoto Y (2009) The actual situation of radiation oncologist. Journal of Niigata Cancer Center Hospital 48: 13-18.

3. Japanese Society for Radiation Oncology website: Let's Become a Radiation Oncologist! http://www.jastro.or.jp/juniordoctor

4. Nishie A, Kakihara D, Nojo T, Nakamura K, Kuribayashi S, et al. (2015) Current radiologist workload and the shortages in Japan: how many full-time radiologists are required. Jpn J Radiol 33: 266-272. [Crossref]

Copyright: (C2017 Tanaka O. This is an open-access article distributed under the terms of the Creative Commons Attribution License, which permits unrestricted use, distribution, and reproduction in any medium, provided the original author and source are credited. 